\title{
Validation of the Spanish Version of the VERITAS-PRN Scale to Assess Adherence to on Demand Regimens in Patients with Hemophilia
}

\author{
Ana Torres-Ortuño $\mathbb{i D}^{\prime}$ \\ Rubén Cuesta-Barriuso (iD ${ }^{2,3}$ \\ Joaquín Nieto-Munuera' \\ Álvaro Castiello-Munuera' \\ José Antonio López-Pina $\mathbb{D}^{4}$ \\ 'Department of Psychiatry and Social \\ Psychology, University of Murcia, Murcia, \\ Spain; ${ }^{2}$ Department of Physiotherapy, \\ University of Murcia, Murcia, Spain; \\ ${ }^{3}$ Royal Foundation Victoria Eugenia, \\ Madrid, Spain; ${ }^{4}$ Department of Basic \\ Psychology and Methodology, University \\ of Murcia, Murcia, Spain
}

Introduction: The episodic or on-demand administration of clotting factor concentrates in hemophilia patients in the event of hemorrhage is employed to restore hemostasis. Adherence to on-demand treatments needs to be assessed in order to improve patient management, avoiding adverse effects and serious clinical complications.

Aim: To validate the Spanish version of the treatment adherence scale in patients with hemophilia, namely, Validated Hemophilia Regimen Treatment Adherence Scale-PRN (VERITAS-PRN).

Methods: Eighty-five patients were recruited in three hemophilia patient associations in Spain. The VERITAS-PRN scale was adapted through a back-translation process from English to Spanish. A native Spanish bilingual translator translated the scale from English to Spanish, and subsequently another native English bilingual translator translated the scale from Spanish to English. The scale was applied twice (two months apart) to assess test-retest reliability.

Results: Internal consistency reliability was slightly lower in the VERITAS-PRN in Spanish $(0.80)$ versus the English version (0.85). There were no differences $(p>0.05)$ between the means of the dimensions or in the total scores between the sample of patients in Spain and the USA. The test-retest reliability coefficient of the scores on the total scale was 0.80 [CI, 0.74-0.86]. The test-retest reliability coefficient was greater than 0.90 in all subscales.

Conclusion: The Spanish version of VERITAS-PRN has high consistency and empirical validity. This scale is useful for assessing the degree of adherence to treatment in adult patients with hemophilia following episodic infusion treatment.

Keywords: hemophilia, adherence, validity, reliability, on-demand treatment, VERITASPRN

\section{Introduction}

Hemophilia is a genetic disease linked to the $\mathrm{X}$ chromosome, characterized by the absence or deficit of any of the blood clotting factors. Depending on the missing factor, there are two types of hemophilia: hemophilia A (factor VIII deficiency) and hemophilia B (factor IX deficiency). ${ }^{1}$ According to the clotting factor levels in functional plasma, there are three categories of hemophilia severity: mild (5-40\% FVIII), moderate $(1-5 \%)$ and severe $(<1 \%){ }^{2}$ The latter are characterized by bleeding in the musculoskeletal system, secondary to mild or even spontaneous trauma.

Hemarthrosis is the main clinical manifestation in patients with hemophilia, localized mainly in knees, elbows and ankles. ${ }^{3}$ The recurrence of joint bleeds leads
Correspondence: Rubén Cuesta-Barriuso Department of Physiotherapy, University of Murcia, Murcia, Spain

Tel +34868887286

Email ruben.cuestab@gmail.com 
to a swelling of the synovial membrane, causing degenerative and progressive joint damage, what is known as hemophilic arthropathy. ${ }^{4}$

The pharmacological treatment of patients with hemophilia requires the intravenous administration of the missing clotting factor using two therapeutic options. ${ }^{5}$ Prophylactic treatment is the periodic administration of clotting factor concentrates (CFCs), while the episodic regimen involves applying the treatment at the onset of a bleeding episode.

Prophylactic treatment represents the gold standard in the prevention of hemophilic arthropathy. ${ }^{6}$ However, implementation of this treatment regimen faces several drawbacks. Venous access, adherence of these patients to the continuous administration of CFCs in the absence of bleeding, the development of inhibitors and the high cost of CFCs represent limitations for the widespread use of prophylactic treatment. ${ }^{7}$ Since there are no guidelines on treatment administration, episodic treatment becomes hindered and entirely relies on each patient's individual responsibility. In fact, patients with hemophilia following on-demand treatment exhibit lower adherence to treatment regimens, with a higher annual bleeding rate and a poorer quality of life. $^{8}$

The patient's adherence to the treatment regimen prescribed by the hematologist and their proper understanding of the need and implications of missing clotting factor replacement are crucial for a successful treatment. ${ }^{9}$ Adherence to treatment in hemophilia requires immediate action in the event of bleeding episodes and being familiar with the different dosages of FVIII/FIX concentrates depending on the severity and evolution of each event. ${ }^{10}$

The use of validated measuring instruments is essential to estimate and approach adherence problems in patients with hemophilia. The validated Hemophilia Regimen Treatment Adherence Scale - On-Demand (VERITASPRN) was designed as a clinical tool and research instrument to establish treatment adherence in patients with hemophilia following on-demand treatment. ${ }^{11}$ This scale has shown good internal consistency in its total score $(\alpha=$ $0.85)$, with excellent test-retest reliability in the subscales $(\mathrm{P}<0.001)$.

The aim of this study is to validate the VERITAS-Pro questionnaire in Spanish for its use in the assessment of adherence to treatment by patients with hemophilia on an on-demand treatment regimen with FVIII/FIX concentrates.

\section{Methods}

\section{Study Design}

Cross-sectional study with a group of patients with hemophilia aged 15 to 64 years.

\section{Patients}

Eighty-five patients with hemophilia were recruited. The patients were recruited from various Hemophilia patient Associations in Spain (Madrid, Murcia and Malaga). Those who met the inclusion criteria were verbally informed of the study objectives. They were provided with a copy of the study objectives (cover letter) by the researchers in a face-to-face meeting, and subsequently signed the informed consent document. They were administered the VERITAS-PRN questionnaire together with a record of sociodemographic and clinical characteristics. The inclusion criteria were: patients diagnosed with hemophilia A or B; over 15 years of age; and following ondemand treatment with FVIII/FIX concentrates.

\section{Ethics and Consent to Participate}

All patients signed an informed consent document, according to the Declaration of Helsinki, after receiving the study information from the head researcher. The study was approved by the Ethics Committee of the Catholic University San Antonio de Murcia (id.24/7/15) and the Spanish Agency for Medicines and Health Products (id.16190/RG32244). In the case of hemophilia patients under the age of 18 , their parents signed the informed consent document. Prior to commencing the study, the protocol was registered in an international clinical trials registry (ClinicalTrials.gov ID: NCT02582450).

\section{Measuring Instrument}

VERITAS-PRN consists of 24 items that measure treatment adherence of patients with bleeding disorders treated on episodic infusion regimens in six dimensions: Treatment, Time, Dose, Plan, Remember, and Communicate. Each item contains five categories from 1 to 5 (always or $100 \%$ of the time, often or at least $75 \%$ of the time, sometimes or at least $50 \%$ of the time, rarely of $25 \%$ of the time, never or $0 \%$ of the time). The highest possible adherence score was 24 and the minimum was 120 in the Spanish version. In each dimension, the score ranged from 4 to 20 points, where 4 is maximum adherence and 20 is minimum adherence. 


\section{Procedure}

The VERITAS-PRN was adapted to Spanish using backtranslation. A bilingual English-Spanish translator translated the scale from the original version. Another translator, not acquainted with the former, translated the Spanish version into English. The two English versions, the original one and the version translated from Spanish, were compared to examine consistency between items. The inconsistencies found were resolved by consensus between the research team and the translators.

The scale was applied twice to patients, two months apart, to evaluate test-retest reliability.

\section{Psychometric and Statistical Analysis}

Mean, standard deviation, skewness, kurtosis and homogeneity index for all items were calculated. The quality of the items was tested using [0.3-0.7] rule. ${ }^{12}$ Also, floor and ceiling effect was calculated in order to test the quality of the scores. A floor or ceiling effect exists when more than $15 \%$ of the subjects obtain the minimum or the maximum score. ${ }^{13}$

Internal consistency of the total scores and the different dimensions was calculated with Cronbach's alpha coefficient. Test-retest reliability coefficient was calculated using the Pearson correlation.

Empirical validity was tested with Student's $t$ and oneway Anova tests for all clinical variables shown in Table 1. The effect size was tested with Cohen's d (small ES: 0.20; medium ES: 0.50; large ES: 0.80 ) or with $\omega^{2}$ (small ES: 0.01; medium ES: 0.06; ES large: 0.14).

\section{Results}

The age of patients was between 15 and 64 years (mean = 44.1; $\mathrm{SD}=10.7$ ). The mean body mass index was 26.4 $(\mathrm{SD}=3.3) \mathrm{kg} / \mathrm{m}^{2}$. Sixty-eight patients $(80.0 \%)$ had a diagnosis of hemophilia A and 17 (20.0\%), of hemophilia $\mathrm{B}$, with a mild disease phenotype in 9 cases $(10.6 \%)$, moderate in 39 cases $(45.9 \%)$ and severe in 37 cases $(43.5 \%)$. All patients $(100 \%)$ were receiving on-demand pharmacological treatment with factor VIII/IX concentrates. In addition, 4 patients $(4.7 \%)$ had developed antibodies to FVIII/IX (inhibitor).

On average, patients' dosage of clotting factor concentrate was 2362 IU (SD: 709.4). Two patients (2.4\%) had port-a-catheters for access to a central venous line and 56 $(65.9 \%)$ had a family history of hemophilia. Forty-eight patients (56.5\%) had presented an average of 1.3 (SD: 1.8) bleeding episodes in the six months prior to data collection, with a range of 0 to 8 . Seventy-three $(85.9 \%)$ had a medical diagnosis of hemophilic arthropathy; 13 $(15.3 \%)$ required orthoses to perform daily life activities and 46 (51.80) had HCV, HIV or both co-infections (Table 1).

\section{VERITAS-PRN Scores}

Table 2 presents the descriptive statistics of the complete scale and of each of the dimensions of VERITAS-PRN. No data were missing. There was no ceiling effect, but a floor effect was obtained in the dimensions Treatment (27.1\%), Time (49.4\%), Dose (23.5\%), Plan (22.4\%), and Remember $(51.8 \%)$, as a result of the high adherence of patients to on-demand treatment $($ mean $=40.2 ; \mathrm{SD}=9.0$ ).

The means of dimensions in the sample of patients in Spain were compared against the means in the original North American sample. There were no significant differences $(p>0.05)$ in the dimensions or in the total scores $(p>0.05)$, showing similar adherence levels in both populations. Significant correlations of between 0.31 and $0.46(\mathrm{p}<0.01)$ were obtained between Treatment vs Time, Dose, Plan and Remember, Time vs Plan and Remember, and Plan vs Remember. All other correlations between dimensions were not significant.

\section{Item Analysis}

Table 3 presents the descriptive statistics and the homogeneity index for each item.

An examination of the means and typical deviations of the items showed that the distribution of replies was strongly biased towards greater adherence to treatment, where many categories obtained no frequency whatsoever or a very low one. In addition, most of the items obtained homogeneity indices in the expected range, but Time item 7, Dose item 12, and Communicate items 21, 22, and 23 obtained discrimination indices below 0.30, implying that further efforts are needed to improve the content of each of those items. Items 12 and 23 showed poorer homogeneity as in both cases the higher categories ( 3 and 4) failed to distinguish between patients according to their lower or greater adherence to treatment (Table 4).

\section{Reliability Analysis}

The test and retest reliability coefficient of the scores on the total scale was 0.80 [CI, 0.74-0.86], although slightly below the reliability coefficient obtained in the US sample (0.85) (Table 5) 
Table I Sociodemographic and Clinical Characteristics of the Patients

\begin{tabular}{|c|c|c|c|c|c|}
\hline & $\mathbf{n}$ & $\%$ & $\mathbf{M}$ & SD & $\mathbf{R}$ \\
\hline Age & & & 44.1 & 10.7 & [15-64] \\
\hline Body Mass Index & & & 26.4 & 3.3 & {$[17-38]$} \\
\hline Type of Hemophilia & & & & & \\
\hline Hemophilia A & 68 & 80.0 & & & \\
\hline Hemophilia B & 17 & 20.0 & & & \\
\hline Severity of & & & & & \\
\hline Hemophilia & & & & & \\
\hline Mild & 9 & 10.6 & & & \\
\hline Moderate & 39 & 45.9 & & & \\
\hline Severe & 37 & 43.5 & & & \\
\hline $\begin{array}{l}\text { Development of } \\
\text { inhibitors }\end{array}$ & & & & & \\
\hline Yes & 4 & 4.7 & & & \\
\hline No & 81 & 95.3 & & & \\
\hline $\begin{array}{l}\text { Dose }^{\mathrm{a}} \text { (patients } \\
\text { without inhibitors) }\end{array}$ & & & 2423.6 & 603.15 & {$[1000-4000]$} \\
\hline $\begin{array}{l}\text { Dose }^{\mathrm{b}} \text { (patients with } \\
\text { inhibitors) }\end{array}$ & & & 5.75 & 0.95 & [5.0-7.0] \\
\hline $\begin{array}{l}\text { Port-a-cat } \\
\text { background data }\end{array}$ & & & & & \\
\hline Yes & 2 & 2.4 & & & \\
\hline No & 83 & 97.6 & & & \\
\hline $\begin{array}{l}\text { Family history of } \\
\text { Hemophilia }\end{array}$ & & & & & \\
\hline Yes & 56 & 65.9 & & & \\
\hline No & 29 & 34.1 & & & \\
\hline $\begin{array}{l}\text { Hemarthrosis in the } \\
\text { previous month }\end{array}$ & & & & & \\
\hline Yes & 48 & 56.5 & & & \\
\hline No & 37 & 43.5 & & & \\
\hline $\begin{array}{l}\text { Number of } \\
\text { hemarthrosis }\end{array}$ & & & 1.3 & 1.8 & [0-8] \\
\hline Arthropathy & & & & & \\
\hline Yes & 73 & 85.9 & & & \\
\hline No & 12 & I4.I & & & \\
\hline Orthoses & & & & & \\
\hline Yes & 13 & 15.3 & & & \\
\hline No & 72 & 84.7 & & & \\
\hline Co-infections & & & & & \\
\hline Yes & 46 & 51.8 & & & \\
\hline No & 39 & 45.9 & & & \\
\hline
\end{tabular}

Notes: Dose ${ }^{a}$ : dosing clotting concentrates (international units); Dose ${ }^{\text {b: bypass }}$ agent dosing (Bethesda units).

Abbreviations: $n$, sample size; \%, percent; $M$, mean; SD, standard deviation; $R$, range.
Table 2 Descriptive Statistics of VERITAS-PRN in the Spanish Sample

\begin{tabular}{|l|c|c|c|c|c|c|}
\hline & M & SD & $\begin{array}{c}\text { Floor } \\
\text { Effect } \\
\text { (\%) }\end{array}$ & $\begin{array}{c}\text { Ceiling } \\
\text { Effect } \\
\text { (\%) }\end{array}$ & Min & Max \\
\hline Total & 40.2 & 9.0 & 12 & 0 & 24 & 72 \\
Treatment & 6.0 & 2.6 & 27.1 & 1.2 & 4 & 20 \\
Time & 6.4 & 3.2 & 49.4 & 0 & 4 & 16 \\
Dose & 6.1 & 1.7 & 23.5 & 0 & 4 & 10 \\
Plan & 6.3 & 2.8 & 22.4 & 1.2 & 4 & 20 \\
Remember & 5.1 & 1.6 & 51.8 & 0 & 4 & 12 \\
Communicate & 10.3 & 3.0 & 47 & 0 & 4 & 16 \\
\hline
\end{tabular}

Abbreviations: M, mean; SD, standard deviation; Min, minimum score; Max, maximum score.

Table 3 Descriptive Statistics and Homogeneity Index of the Items of VERITAS-PRN

\begin{tabular}{|c|c|c|c|c|c|}
\hline Items & M & SD & Sk & Ku & IH \\
\hline \multicolumn{6}{|l|}{ Treatment } \\
\hline II & 1.45 & 1.01 & 2.54 & 5.83 & 0.73 \\
\hline 12 & 1.68 & 0.83 & 2.18 & 6.97 & 0.56 \\
\hline 13 & 1.49 & 0.76 & 2.45 & 8.72 & 0.42 \\
\hline 14 & 1.31 & 0.63 & 2.99 & 12.78 & 0.36 \\
\hline \multicolumn{6}{|l|}{ Time } \\
\hline 15 & 1.53 & 0.93 & 1.81 & 3.03 & 0.61 \\
\hline 16 & 4.31 & 1.17 & -1.69 & 1.82 & 0.34 \\
\hline 17 & 4.64 & 0.84 & -2.80 & 8.20 & 0.22 \\
\hline 18 & 1.77 & 1.18 & 1.47 & 1.05 & 0.57 \\
\hline \multicolumn{6}{|l|}{ Dose } \\
\hline 19 & 1.31 & 0.60 & 3.13 & 15.69 & 0.32 \\
\hline 110 & 1.11 & 0.42 & 4.77 & 27.66 & 0.37 \\
\hline III & 1.34 & 0.68 & 2.87 & 10.99 & 0.35 \\
\hline 112 & 3.69 & 1.20 & -0.56 & -0.72 & -0.04 \\
\hline \multicolumn{6}{|l|}{ Plan } \\
\hline 113 & 3.97 & 1.05 & -1.59 & 2.43 & 0.10 \\
\hline 114 & 1.46 & 0.86 & 2.77 & 8.69 & 0.57 \\
\hline 115 & 1.51 & 0.94 & 2.62 & 7.18 & 0.65 \\
\hline 116 & 1.28 & 0.85 & 3.58 & 12.64 & 0.49 \\
\hline \multicolumn{6}{|l|}{ Remember } \\
\hline 117 & 4.77 & 0.59 & -3.85 & 20.03 & 0.38 \\
\hline 118 & 1.36 & 0.98 & 3.14 & 9.13 & 0.43 \\
\hline 119 & 4.83 & 0.38 & -1.77 & 1.14 & 0.18 \\
\hline 120 & 4.71 & 0.48 & -1.28 & $0.4 \mathrm{I}$ & 0.17 \\
\hline \multicolumn{6}{|l|}{ Communicate } \\
\hline $12 \mid$ & 3.05 & 1.08 & -0.21 & -0.82 & 0.13 \\
\hline 122 & 3.00 & 1.24 & 0.00 & -1.01 & 0.27 \\
\hline 123 & 3.20 & 1.07 & 0.26 & -0.94 & -0.08 \\
\hline 124 & 1.39 & 0.80 & 2.55 & 6.93 & 0.37 \\
\hline
\end{tabular}

Abbreviations: M, mean; SD, standard deviation; Sk, skewness; Ku, kurtosis; $\mathrm{IH}$, homogeneity index; $\alpha$, alpha coefficient. 
Table 4 Mean, Standard Deviation of the Total Score in Each Category of VERITAS-PRN

\begin{tabular}{|c|c|c|c|c|c|}
\hline Categories & I & 2 & 3 & 4 & 5 \\
\hline Item & $M(S D)$ & $M(S D)$ & $M(S D)$ & $M(S D)$ & $M(S D)$ \\
\hline \multicolumn{6}{|l|}{ Treatment } \\
\hline II & $36.7(5.5)$ & $45.4(6.1)$ & $51.3(2.1)$ & $47.5(0.7)$ & $67.3(3.7)$ \\
\hline 12 & $37.2(8.6)$ & $40.2(5.2)$ & $51.5(0.7)$ & - & $68.7(2.9)$ \\
\hline 13 & $38.5(8.9)$ & $40.3(5.2)$ & $51.5(0.7)$ & - & $69.5(3.5)$ \\
\hline 14 & $38.6(8.3)$ & $42.8(8.5)$ & $51.5(0.7)$ & - & $67.0(-)$ \\
\hline \multicolumn{6}{|l|}{ Time } \\
\hline 15 & $36.7(5.6)$ & $44.9(9.9)$ & $47.6(8.3)$ & - & $61.5(6.4)$ \\
\hline 16 & $37.2(7.3)$ & $44.0(9.1)$ & $47.3(5.3)$ & $44.3(8.6)$ & $50.2(14.6)$ \\
\hline 17 & $37.9(7.7)$ & $52.0(8.4)$ & $43.4(8.8)$ & $51.0(-)$ & $42.0(1.4)$ \\
\hline 18 & $35.9(5.4)$ & $41.6(4.8)$ & $52.1(12.3)$ & $46.3(5.3)$ & $57.3(\mid 3.3)$ \\
\hline \multicolumn{6}{|l|}{ Dose } \\
\hline 19 & $38.9(9.0)$ & $42.1(5.7)$ & - & - & $72.0(-)$ \\
\hline 110 & $39.2(8.2)$ & $47.4(9.8)$ & - & $67.0(-)$ & - \\
\hline III & $38.2(7.7)$ & $43.9(9.5)$ & $52.0(-)$ & $67.0(-)$ & $48.0(-)$ \\
\hline 112 & $38.8(\mid 3.3)$ & $41.4(8.4)$ & $39.1(3.6)$ & $42.9(3.7)$ & $40.3(4.1)$ \\
\hline \multicolumn{6}{|l|}{ Plan } \\
\hline 113 & $39.8(12.8)$ & $38.9(5.7)$ & $42.5(6.8)$ & $51.0(15.1)$ & $44.8(7.7)$ \\
\hline 114 & $37.1(6.8)$ & $43.5(7.5)$ & - & $67.0(-)$ & $61.0(7.2)$ \\
\hline 115 & $37.1(6.6)$ & $42.0(5.5)$ & - & $67.0(-)$ & $63.8(8.1)$ \\
\hline 116 & $38.0(6.5)$ & $51.9(12.9)$ & $47.0(-)$ & $46.0(-)$ & $61.0(7.2)$ \\
\hline \multicolumn{6}{|l|}{ Remember } \\
\hline 117 & $38.5(8.1)$ & $46.9(7.9)$ & $37.0(-)$ & - & $67.0(-)$ \\
\hline 118 & $37.9(6.5)$ & $47.0(10.1)$ & $63.0(-)$ & - & $54.2(14.1)$ \\
\hline 119 & $29.3(8.9)$ & $44.4(8.2)$ & - & - & - \\
\hline 120 & $38.8(8.4)$ & $44.0(9.8)$ & $36.0(-)$ & - & - \\
\hline \multicolumn{6}{|l|}{ Communicate } \\
\hline 121 & $39.4(15.2)$ & $36 . I(9.6)$ & $40.1(6.6)$ & $42.7(8.4)$ & $43.2(3.1)$ \\
\hline 122 & $34.6(11.7)$ & $37.4(6.9)$ & $40.5(8.5)$ & $41.9(7.3)$ & $47.2(9.2)$ \\
\hline 123 & $40.9(14.2)$ & $40.4(12.3)$ & $38.3(4.7)$ & $40.8(5.8)$ & 49.5(4.9) \\
\hline 124 & $38.0(7.7)$ & $43.4(7.5)$ & $50.0(1.4)$ & $60.7(12.7)$ & $38.0(-)$ \\
\hline
\end{tabular}

Abbreviations: $M$, mean; $S D$, standard deviation; $r_{j x}$, discrimination index; $\alpha$, alpha coefficient.

Table 5 Reliability Coefficients of Scores of the VERITAS-PRN in Spain and USA

\begin{tabular}{|l|c|c|c|c|}
\hline \multirow{2}{*}{ Items } & \multicolumn{2}{|c|}{ Spain } & \multicolumn{2}{c|}{ USA } \\
\cline { 2 - 5 } & \multicolumn{2}{|c|}{ Alpha } & Pearson & Alpha \\
\cline { 2 - 5 } & Test & Retest & Test-Retest & Test \\
\hline Treatment & 0.78 & 0.68 & 0.93 & 0.78 \\
Time & 0.76 & 0.85 & 0.96 & 0.76 \\
Dose & 0.22 & 0.35 & 0.95 & 0.63 \\
Plan & 0.42 & 0.71 & 0.94 & 0.8 \\
Remember & 0.66 & 0.49 & 0.94 & 0.66 \\
Communicate & 0.80 & 0.60 & 0.91 & 0.80 \\
Total & 0.85 & 0.80 & 0.94 & 0.85 \\
\hline
\end{tabular}

The scores in the Time dimension obtained the same reliability coefficient in both Spanish and US samples (0.76), while in the Treatment (0.78 vs 0.74$)$, and Plan ( 0.76 vs 0.48$)$ dimensions, the reliability coefficient of the scores was higher in the Spanish sample than in the US sample. The reliability coefficient of the scores was higher in the US sample for the Dose (0.22 vs 0.63$)$, Remember (0.42 vs 0.66) and Communicate (0.66 vs 0.80$)$ dimensions. The low reliability coefficient obtained for Dose in the Spanish sample was significant, showing the need for an in-depth analysis of the operation of this dimension in the Spanish version of the VERITAS-PRN scale. 
The test-retest reliability, evaluated two months after the first application, showed a high stability of the scores in both applications where the reliability coefficient was not below 0.90 in any of the subscales (Table 5).

\section{Empirical Validity}

No significant differences were found in any of the dimensions or in the total score based on the type of hemophilia, the development of inhibitors or the use of orthoses (Table 6).

Patients with moderate or severe types showed greater adherence to treatment than patients with a mild phenotype $(\mathrm{F}[2,85]=11.63 ; \mathrm{p}<0.001 ; \mathrm{ES}=0.43)$, better controlling the Time dimension $(\mathrm{F}[2,85]=12.72 ; \mathrm{p}<0.001 ; \mathrm{ES}=$ 0.46). However, patients with mild and severe phenotypes planned better than patients with moderate severity ( $\mathrm{F}[2$, $85]=7.59 ; \mathrm{p}=0.001 ; \mathrm{ES}=0.32$ ). Overall, adherence to treatment in all dimensions was greater in patients with moderate and severe types than in patients with a mild phenotype $(\mathrm{F}[2,85]=10.99 ; \mathrm{p}<0.001 ; \mathrm{ES}=0.42)$.
Patients with hemarthrosis were better with treatment planning $(\mathrm{t}[45.70]=-2.50, \mathrm{p}<0.05, \mathrm{ES}=-0.55)$. On the other hand, patients with arthropathy showed greater adherence to treatment $(\mathrm{t}[11.24]=-2.98 ; \mathrm{p}<0.05 ; \mathrm{ES}=$ $-0.93)$, time management $(\mathrm{t}[83]=-7.03 ; \mathrm{p}<0.001$; ES $=-2.19)$ and in general $(\mathrm{t}[12.55]=-6.07 ; \mathrm{p}<0.001$; ES $=-1.89$ ). In addition, patients with coinfections proved to be better at remembering doses $(\mathrm{t}[76.64]=-3.29 ; \mathrm{p}=$ $0.002 ; \mathrm{ES}=-0.72$ ).

\section{Discussion}

The aim of this study was to validate the VERITAS-PRN questionnaire in Spanish, to assess adherence to drug treatment in patients with hemophilia following ondemand treatment. The VERITAS-PRN scale presents good psychometric properties to measure adherence to episodic regimens in hemophilia. Almost all items obtained homogeneity indices within the expected range, and the reliability coefficients of the dimensions were acceptable except in the Dose dimension. The stability of

Table 6 Mean (SD) of the Dimensions of VERITAS-PRN

\begin{tabular}{|c|c|c|c|c|c|c|c|}
\hline & (I) & (2) & (3) & (4) & (5) & (6) & (7) \\
\hline \multicolumn{8}{|l|}{ Type of Hemophilia } \\
\hline Hemophilia A & $6.0(2.8)$ & 6.3(3.2) & $6.2(1.8)$ & 6.3(2.6) & $5.2(1.6)$ & $10.6(2.9)$ & $40.4(9.6)$ \\
\hline Hemophilia B & $5.9(0.9)$ & $6.7(3.3)$ & $6.0(1.4)$ & $6.6(3.8)$ & $4.8(1.5)$ & $9.1(2.9)$ & 39.(5.9) \\
\hline \multicolumn{8}{|l|}{ Severity } \\
\hline Low & $9.4(5.6)^{* * *}$ & $\begin{array}{c}10.3(3.3) \\
* * *\end{array}$ & $6.3(1.9)$ & $5.5(1.4)^{* * *}$ & $6.2(1.9)$ & $10.3(3.1)$ & $52.0(\mid 3.1)^{* * * *}$ \\
\hline Moderate & $5.5(1.8)$ & $5.2(2.6)$ & $6.1(1.8)$ & $6.4(3.2)$ & $4.8(1.5)$ & $10.3(2.9)$ & $38.2(7.8)$ \\
\hline Severe & $5.7(1.3)$ & $6.7(2.9)$ & $6.1(1.6)$ & $5.5(1.4)$ & $5.1(1.5)$ & $10.3(3.1)$ & $39.4(6.9)$ \\
\hline \multicolumn{8}{|l|}{ Inhibitors } \\
\hline Yes & $6.8(2.5)$ & $8.8(3.4)$ & $5.0(0.8)$ & $5.5(1.9)$ & $6.3(2.9)$ & $9.8(4.3)$ & $42.0(12.5)$ \\
\hline No & $5.9(2.6)$ & $6.3(3.1)$ & $6.2(1.7)$ & $6.4(2.9)$ & $5.0(1.5)$ & $10.3(2.9)$ & $40.1(8.9)$ \\
\hline \multicolumn{8}{|l|}{ Hemarthrosis } \\
\hline Yes & $5.8(1.7)$ & $6.5(3.0)$ & $6.0(1.7)$ & $5.6(1.6)^{*}$ & $4.9(1.3)$ & $10.6(3.2)$ & $29.4(7.2)$ \\
\hline No & $6.2(3.4)$ & $6.2(3.5)$ & $6.3(1.8)$ & $7.2(3.8)$ & $5.2(1.8)$ & $9.9(2.7)$ & $41.2(10.9)$ \\
\hline \multicolumn{8}{|l|}{ Arthropathy } \\
\hline Yes & $5.4(1.2)^{* * * *}$ & $5.6(2.6)^{* * *}$ & $6.1(1.7)$ & $5.6(1.5)$ & $4.9(1.3)$ & $10.2(2.9)$ & $37.7(6.2)^{* * *}$ \\
\hline No & $9.6(4.9)$ & II.2(2.2) & $6.4(2.0)$ & $10.4(5.1)$ & $6.4(2.5)$ & II.0(3.4) & $55.0(9.5)$ \\
\hline \multicolumn{8}{|l|}{ Infections } \\
\hline Yes & $5.7(2.3)$ & $6.1(3.2)$ & $6.4(1.8)$ & $6.5(2.8)$ & $4.5(I . I)^{* *}$ & $10.3(3.4)$ & $39.4(8.4)$ \\
\hline No & $6.2(2.8)$ & $6.7(3.2)$ & $5.9(1.6)$ & $6.2(2.9)$ & $5.5(1.8)$ & $10.3(2.6)$ & $40.9(9.5)$ \\
\hline \multicolumn{8}{|l|}{ Orthoses } \\
\hline Yes & $5.2(1.3)$ & $6.5(3.0)$ & $5.7(1.7)$ & $5.2(1.5)$ & $5.0(1.4)$ & $9.2(3.2)$ & $38.7(8.7)$ \\
\hline No & $6.1(2.7)$ & $6.4(3.2)$ & $6.2(1.7)$ & $6.5(3.0)$ & $5.1(1.6)$ & $10.5(2.9)$ & $40.8(9.0)$ \\
\hline
\end{tabular}

Notes: (I) Treatment; (2) time; (3) dose; (4) plan; (5) remember; (6) communicate; (7) total. ${ }^{*} \mathrm{p}<0.05$; ${ }^{* *} \mathrm{p}<0.01$; ${ }^{* * *} \mathrm{p}<0.00 \mathrm{I}$. 
the scores was also very high, suggesting that this scale can be safely used to monitor the early management of bleeding episodes in order to control adherence in patients with hemophilia on episodic regimens.

Almost half of patients with hemophilia report a late handling of bleeding episodes, failing to act in the first hours of bleeding. ${ }^{14} \mathrm{Up}$ to $40 \%$ of these patients report that they do not strictly follow the therapeutic guidelines prescribed by their hematologist of reference in hemophilia for the treatment of acute bleeding. ${ }^{15,16}$ This may be due to the lack of awareness about the importance of early treatment of bleeding episodes ${ }^{17}$ and the changes in the dosage of clotting factor concentrates made by patients especially in the first hours of acute bleeding. Our study observed a low reliability coefficient in the Dose domain. The bleeding experiences, the memory of previous bleeds and intense pain can be factors that push patients to significantly increase the dose of factors in the first hours of development.

Poor adherence to CFC treatment may favor the recurrence of hemarthrosis or hematomas, further complicating the patient's clinical musculoskeletal status, leading to the development of hemophilic arthropathy at an early age. ${ }^{18}$ Although prophylactic treatment is increasingly widespread, it is essential to understand and measure the adherence of patients on episodic regimens, after a hemorrhagic process. A proper therapeutic approach to acute hemorrhages is essential in the development and evolution of joint damage in these patients. ${ }^{19}$ Multidisciplinary teams working with patients with hemophilia should include the use of scales such as VERITAS-PRN to initially establish the degree of adherence of on-demand treatment patients and, subsequently, address those aspects that can be improved in the prevention of future complications in case of more severe bleeding episodes.

Patients with mild hemophilia, having a lower incidence of bleeding, may present poorer values in terms of adherence to treatment. The absence of a history of hemarthrosis and previous bleeding events may be a risk factor that reduces adherence to treatment in these patients. Accordingly, the adherence of patients with mild hemophilia needs to be evaluated, despite their low incidence of bleeding and the lower overall risk, compared to patients with severe and moderate hemophilia.

\section{Limitations of the Study}

The largest limitation of the study is the small sample size used, which means that future studies should use a larger sample to corroborate the difficulties encountered in measuring the Dose and Remember dimensions.

The increasingly widespread use of prophylactic treatment makes it difficult to recruit hemophilia patients with a severe phenotype following on-demand treatment. To obtain a representative sample, the recruitment was carried out in different regions of Spain with patients treated at varying types of hemophilia hospitals (from internationally known hemophilia units to small regional hospitals). The large differences in terms of access to information and medical services may be a limitation as patients with varying hemophilia management and care approaches are included in the study. Similarly, the inclusion of patients with inhibitors and different phenotypes of hemophilia may be a limitation regarding the homogeneity in replies.

\section{Conclusions}

The Spanish version of VERITAS-PRN presents high levels of consistency and empirical validity. This measuring instrument is useful for assessing the degree of adherence to medical treatment by adult patients with hemophilia following on-demand treatment. Future clinical studies evaluating treatment adherence in adult patients with hemophilia on episodic infusion treatments should confirm the findings of this study in terms of the validity of the VERITAS-PRN questionnaire.

\section{Data Sharing Statement}

The dataset used is available from the corresponding author upon request. Encoded data without personal identification shall solely be provided to those directly requesting same for scientific purposes.

\section{Ethics and Consent to Participate}

The study was approved by the Ethics Committee of the Universidad Católica San Antonio of Murcia (id.24/7/15) and the Spanish Agency for Medicines and Health Products (id.16190/RG32244).

\section{Acknowledgments}

The authors are especially grateful to Regional Murciana Association of Hemophilia, Association of Hemophilia of Burgos, Gallega Association of Hemophilia and the Spanish Federation of Hemophilia, for their help in recruitment of the sample. 


\section{Funding}

The authors wish to thank Medtep Inc., promoter of the u-patient study. Source of financial support: Grifols Inc., provided funding for this study in accordance with international guidelines on Good Publication Practice.

\section{Disclosure}

The authors certify that there are no conflicts of interest with any financial organization regarding the material discussed in the manuscript.

\section{References}

1. Molho P, Rolland N, Lebrun T, et al. Epidemiological survey of the orthopaedic status of severe haemophilia A and B patients in France. Haemophilia. 2000;6(1):23-32. doi:10.1046/j.1365-2516.20 00.00358.x

2. White GC, Rosendaal F, Aledort LM, et al. Definitions in hemophilia. Recommendation of the scientific subcommittee on factor VIII and factor IX of the scientific and standardization committee of the International Society on Thrombosis and Haemostasis. Thromb Haemost. 2001;85(3):560. doi:10.1055/s-0037-1615621

3. Srivastava A, Brewer AK, Mauser-Bunschoten EP, et al.; Treatment Guidelines Working Group on Behalf of The World Federation of Hemophilia. Guidelines for the management of hemophilia. Haemophilia. 2013;19:e1-e47.

4. Soucie JM, Cianfrini C, Janco RL, et al. Joint range-of-motion limitations among young males with hemophilia: prevalence and risk factors. Blood. 2004;103(7):2467-2473. doi:10.1182/blood-2003-051457

5. Berntorp E, Shapiro AD. Modern haemophilia care. Lancet. 2012;379 (9824):1447-1456. doi:10.1016/S0140-6736(11)61139-2

6. Manco-Johnson MJ, Abshire TC, Shapiro AD, et al. Prophylaxis versus episodic treatment to prevent joint disease in boys with severe hemophilia. N Engl J Med. 2007;357(6):535-544. doi:10.1056/ NEJMoa067659

7. Windyga J. Is continuous low-dose prophylaxis superior to on-demand treatment for patients with hemophilia? Semin Thromb Hemost. 2016;42(5):533-540. doi:10.1055/s-0036-1579639
8. Zappa S, McDaniel M, Marandola J, Allen G. Treatment trends for haemophilia A and haemophilia B in the United States: results from the 2010 practice patterns survey. Haemophilia. 2012;18(3):e140e153. doi:10.1111/j.1365-2516.2012.02770.x

9. Osterberg L, Blaschke T. Adherence to medication. N Engl J Med. 2005;353(5):487-497. doi:10.1056/NEJMra050100

10. Tran DQ, Barry V, Antun A, Ribeiro M, Stein S, Kempton CL. Physician trust and depression influence adherence to factor replacement: a Single-Centre Cross-Sectional Study. Haemophilia. 2017;23 (1):98-104. doi:10.1111/hae.13078

11. Duncan NA, Kronenberger WG, Roberson CP, Shapiro AD. VERITAS-PRN: a new measure of adherence to episodic treatment regimens in haemophilia. Haemophilia. 2010;16(1):47-53. doi:10.1111/j.1365-2516.2009.02094.x

12. Streiner DL, Norman GR. Health Measurement Scales: A Practical Guide to Their Development and Use. 4rd ed. Oxford: Oxford University Press; 2008.

13. McHorney CA, Tarlov AR. Individual-patient monitoring in clinical practice: are available health status surveys adequate? Qual Life Res. 1995;4(4):293-307. doi:10.1007/BF01593882

14. Nazzaro AM. Where are we: preliminary results of the national hemophilia foundation's prevention program baseline survey of health knowledge, attitudes, and behaviors in the hemophilia population. HemAware. 2000;20-5:24-25.

15. Lindvall K, Colstrup L, Wollter IM, et al. Compliance with treatment and understanding of own disease in patients with severe and moderate haemophilia. Haemophilia. 2006;12(1):47-51. doi:10.1111/ j.1365-2516.2006.01192.x

16. Hacker MR, Geraghty S, Manco-Johnson M. Barriers to compliance with prophylaxis therapy in haemophilia. Haemophilia. 2001;7 (4):392-396. doi:10.1046/j.1365-2516.2001.00534.x

17. Saxena K. Barriers and perceived limitations to early treatment of hemophilia. J Blood Med. 2013;4:49-56. doi:10.2147/JBM.S43734

18. McLaughlin JM, Witkop ML, Lambing A, Anderson TL, Munn J, Tortella B. Better adherence to prescribed treatment regimen is related to less chronic pain among adolescents and young adults with moderate or severe haemophilia. Haemophilia. 2014;20 (4):506-512. doi:10.1111/hae.12360

19. Pasta G, Mancuso ME, Perfetto OS, Solimero LP. Radiosynoviorthesis in children with haemophilia. Hämostaseologie. 2009;29(1):S62-64. doi:10.1055/s-0037-1621502

\section{Publish your work in this journal}

Patient Preference and Adherence is an international, peer-reviewed, open access journal that focusing on the growing importance of patient preference and adherence throughout the therapeutic continuum. Patient satisfaction, acceptability, quality of life, compliance, persistence and their role in developing new therapeutic modalities and compounds to optimize clinical outcomes for existing disease states are major areas of interest for the journal. This journal has been accepted for indexing on PubMed Central. The manuscript management system is completely online and includes a very quick and fair peer-review system, which is all easy to use. Visit http:// www.dovepress.com/testimonials.php to read real quotes from published authors. 